\title{
Development of an Efficient Hull Form Design Exploration Framework
}

\author{
Shinkyu Jeong ${ }^{1}$ and Hyunyul Kim ${ }^{2}$ \\ ${ }^{1}$ Department of Mechanical Engineering, Kyunghee University, Yongin 446-701, Republic of Korea \\ ${ }^{2}$ Department of Computational and Data Science, George Mason University, Fairfax, VA 22030, USA \\ Correspondence should be addressed to Shinkyu Jeong; icarus@khu.ac.kr
}

Received 16 April 2013; Accepted 24 June 2013

Academic Editor: Ming Li

Copyright (c) 2013 S. Jeong and H. Kim. This is an open access article distributed under the Creative Commons Attribution License, which permits unrestricted use, distribution, and reproduction in any medium, provided the original work is properly cited.

\begin{abstract}
A high-efficiency design exploration framework for hull form has been developed. The framework consists of multiobjective shape optimization and design knowledge extraction. In multiobjective shape optimization, a multiobjective genetic algorithm (MOGA) using the response surface methodology is introduced to achieve efficient design space exploration. As a response surface methodology, the Kriging model, which was developed in the field of spatial statistics and geostatistics, is applied. A new surface modification method using shifting method and radial basis function interpolation is also adopted here to represent various hull forms. This method enables both global and local modifications of hull form with fewer design variables. In design knowledge extraction, two data mining techniques - functional analysis of variance (ANOVA) and self-organizing map (SOM) —are applied to acquire useful design knowledge about a hull form. The present framework has been applied to hull form optimization exploring the minimum wave drag configuration under a wide range of speeds. The results show that the present method markedly reduced the design period. From the results of data mining, it is possible to identify the design variables controlling wave drag performances at different speed regions and their corresponding geometric features.
\end{abstract}

\section{Introduction}

In order to find the combination of design variables that generates a hull form with optimal hydrodynamic performance (e.g., drag and seakeeping), a tremendous number of performance evaluations are required. However, it is obvious that an increase in the number of evaluations causes an excessive increase in both the cost and time of the design process. Recently, computational-fluid-dynamics-(CFD-) based optimization techniques have become promising alternative tools in ship hydrodynamic design. To be competitive in the shipbuilding market, a practical and efficient CFD-based ship hydrodynamic optimization tool is essential in the preliminary and early design stages to aid in prompt and accurate design.

CFD-based hull form hydrodynamic optimization consists of a CFD solver that computes the flow field and evaluates the performance of hull form (objective functions), hull form modeling with design variables, and an optimization technique that explores the combination of the design parameters generating the hull form with the optimum performance under the given constraints.

Recently, optimization techniques that mimic the course of biological evolution and the movement of living organisms, which is known as evolutionary algorithms (EAs), have been widely used in the field of ship design. The typical examples of methodology are genetic algorithms (GAs) $[1,2]$ and particle swarm optimization [3]. Lowe and Steel [4] applied a genetic algorithm to search for a hull form satisfying prescribed primary and secondary form parameters within the design space. Tahara et al. [5-7] used a combination of successive quadratic programming and multiobjective genetic algorithm to optimize tanker stern, Delta Catamaran, and so on. Gammon [8] studied hull form optimization of fishing vessels using multiobjective genetic algorithm. Three performance indices for resistance, seakeeping, and stability were considered in this multiobjective optimization. In particular, automatic selection of a few Pareto optimal solutions was 
developed so that the designer can easily choose the more favorable candidates. More recently, Knight et al. [9] applied particle swarm optimization to incorporate uncertainty in parameters into the optimization of a planning craft.

However, the number of CFD runs required in these methods is too large. Furthermore, if these methods are combined with a time-consuming high fidelity CFD solver, it is difficult to complete the design process in reasonable time. Therefore, it is very important to either have a highly efficient CFD tool or to use an accurate approximation model (e.g., response surface) to partially replace the CFD runs for the CFD-based optimization tool to be useful in practical ship design.

The response surface method is a well-established approach for creating approximation models based on sample data that can be obtained from physical experiments and computer simulations [10]. A major advantage of using the response surface method in CFD-based design optimization is to reduce the computational costs associated with the CFD runs. A given optimization problem can be approximated by the response surface method with smooth functions that can improve computational efficiency in the optimization process. The response surface method has been applied to many CFD-based aerodynamics optimization problems, including a centrifugal fan and transonic airfoil, and this approach was shown to be quite efficient in exploring the design space $[11,12]$.

In the present study, the Kriging response surface model [13-15] is implemented in the CFD-based ship hydrodynamics optimization tool to provide the objective function values associated with the hydrodynamics performance efficiently. The Kriging model has a sufficient flexibility to represent the nonlinear and multimodal functions which are often appeared in hydrodynamic performances. Another merit of Kriging model is that it predicts not only function value but also the uncertainty of the estimated function value. Based on both the function value and its uncertainty, it is possible to improve the accuracy of Kriging model during the design process.

In this study, the Kriging model based multiobjective shape optimization method is applied to the optimal hull form design to minimize wave drag over a wide range of design speeds. For the modeling of hull form, a surface modification method using shifting method and radial basis function interpolation is introduced. The shifting method makes it possible to realize a smooth junction of the modified portion with the original design. The radial basis function is used for the local modifications of hull form. Thus, these methods enable both global and local modification of hull form with fewer design variables. In design knowledge extraction, two data mining techniques-functional analysis of variance (ANOVA) [16] and self-organizing map (SOM) [17]—are applied to acquire useful design knowledge about a hull form. Functional analysis of variance (ANOVA) shows effect of each design variable on objective functions. Self-organizing map (SOM) identifies the design variables controlling the trade-off between high-speed wave drag and low-speed wave drag. Numerical results show that the design exploration framework not only can explore superior hull forms within a short computational time but also supplies the insight of the effect of each design variable on the drag performance at different design speeds.

The remainder of this paper is organized as follows. In the next two sections, design problem definition and design exploration used in this study will be introduced, respectively. This will be followed by results of shape optimization and design knowledge extraction.

\section{Design Problem Definition}

2.1. Hull Form Definition and Design Variables. An accurate and effective hull surface representation and modification technique plays an important role in the CFD-based hull form hydrodynamic optimization. In the present study, the Series60 (block coefficient: $\left.C_{b}=0.6\right)[18,19]$ hull model is adopted as the initial hull form and modified to represent a new hull form using the shifting method [20-22] and the radial basis function (RBF) interpolation [23] during shape optimization. These methods make it possible to represent both global and local modifications of hull form with smaller design variables.

First, the sectional area curve of the initial hull form is modified using the shifting method, which moves the stations of the initial hull form along the longitudinal direction. The amount of movement is determined by comparing the modified sectional area curve with the original curve. In this study, the sectional area curve of the initial hull form is modified using (1a) and (1b):

$$
\begin{gathered}
f^{n}(x)=f^{0}(x)+g\left(x, \alpha_{1}, \alpha_{2}\right) \\
g\left(x, \alpha_{1}, \alpha_{2}\right) \\
\quad \begin{cases}\alpha_{1} \sqrt{0.5\left(1-\cos \left(2 \pi \frac{x-x_{1}}{\alpha_{2}-x_{1}}\right)\right)}, & x_{1} \leq x \leq \alpha_{2}, \\
-\alpha_{1} \sqrt{0.5\left(1-\cos \left(2 \pi \frac{x-\alpha_{2}}{\alpha_{2}-x_{2}}\right)\right)}, & \alpha_{2} \leq x \leq x_{2}, \\
0, & \text { elsewhere, }\end{cases}
\end{gathered}
$$

where $f^{n}(x)$ and $f^{0}(x)$ denote the sectional area curve of the new and initial hull forms, respectively, [21, 22]. Thus, $g\left(x, \alpha_{1}, \alpha_{2}\right)$ indicates the amount of shape modification at position $x$, and $x_{1}$ and $x_{2}$ are fixed points.

As shown in Figure 1, the slope of the sectional area curve is defined by $\alpha_{1}$ and the location of the fixed station is controlled using $\alpha_{2}$. By changing the parameters $\alpha_{1}$ and $\alpha_{2}$, various sectional area curves can be obtained. In comparison with the spline polynomial representation, this formulation can prevent the generation of unrealistic hull forms associated with oscillation of the new sectional area curve. In this study, the sectional area curves of both fore and aft bodies are modified simultaneously using four design variables: entrance angle $\left(\alpha_{1 f}\right)$, run angle $\left(\alpha_{1 a}\right)$, fore-body variation $\left(\alpha_{2 f}\right)$, and aft-body variation $\left(\alpha_{2 a}\right)$.

Once the modified sectional area curve is obtained, local modification of the hull form is performed using $\mathrm{RBF}$ interpolation. In this study, the displacement of point 
TABLE 1: Summary of optimization applications.

\begin{tabular}{lccc}
\hline Design variable & Definition & Modification & Range \\
\hline DV1 & Fore-body variation & $\alpha_{2 f}$ & $0.30 \pm 0.2$ \\
DV2 & Entrance angle & $\alpha_{1 f}$ & $\pm 0.5^{-2}$ \\
DV3 & Aft-body variation & $\alpha_{2 a}$ & $-0.30 \pm 0.2$ \\
DV4 & Run angle & $\alpha_{1 a}$ & $\pm 0.5^{-2}$ \\
DV5-DV12 & Local body variation & $x$-coordinate of RBF 1-8 & $0.050 \pm 0.005$ \\
DV13 & Stem line variation & $y$-coordinate of RBF 9 & $0.493-0.500$ \\
DV14 & Stem line variation & $y$-coordinate of RBF 10 & $0.495-0.501$ \\
DV15 & Stem line variation & $y$-coordinate of RBF 11 & $0.499-0.507$ \\
DV16 & Stem line variation & $y$-coordinate of RBF 12 & $0.505-0.520$ \\
\hline
\end{tabular}

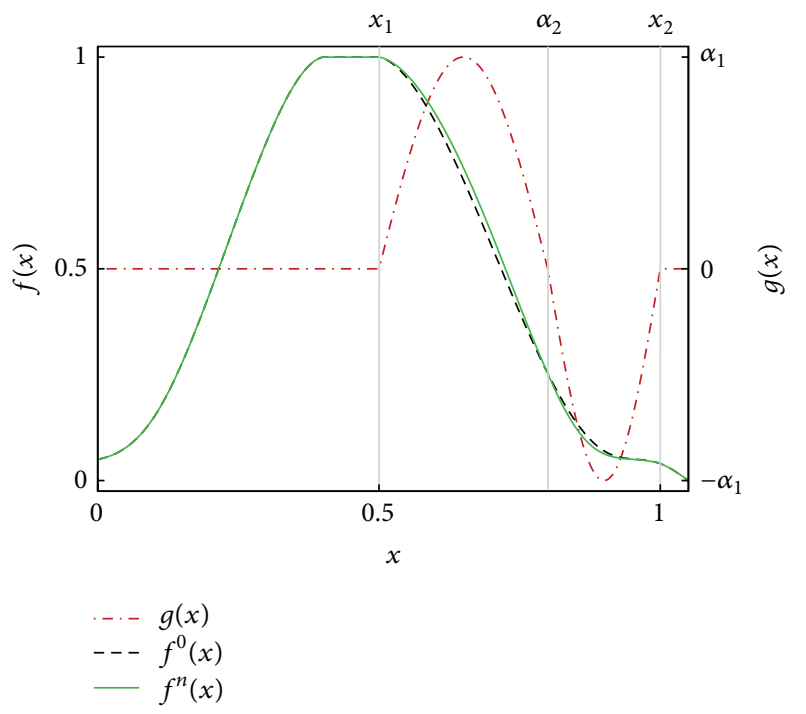

FIGURE 1: Section area curves and shape function.

$\mathbf{x}=(x, y, z)$ on the hull form is described by the following $\mathrm{RBF}$ interpolation:

$$
s(\mathbf{x})=\sum_{j=1}^{N} \lambda_{j} \varphi\left(\left\|\mathbf{x}-\mathbf{x}_{j}\right\|\right)+p(\mathbf{x}),
$$

where $\mathbf{x}_{j}=\left(x_{j}, y_{j}, z_{j}\right)$ is the center of the RBF, $N$ is the number of control nodes (centers), and $\varphi$ is a given RBF with respect to the Euclidean distance $\|\mathbf{x}\|$. In the present study, the $\operatorname{RBF} \varphi$ in (2) is defined in terms of Wendland's C2 function as follows:

$$
\varphi(\|\mathbf{x}\|)=(1-\|\mathbf{x}\|)^{4}(4\|\mathbf{x}\|+1),
$$

and the polynomial $p$ in (2) is defined in terms of the linear polynomial to recover the rotation and translation as follows:

$$
p(\mathbf{x})=c_{1}+c_{2} x+c_{3} y+c_{4} z
$$

The coefficients $\lambda_{j}$ in (2) and $c_{j}$ in (4) are determined by the interpolation conditions

$$
s\left(\mathbf{x}_{j}\right)=f_{j}, \quad j=1, \ldots, N
$$

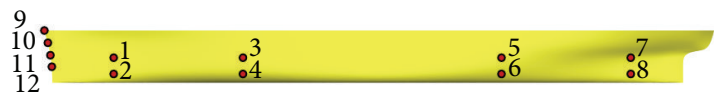

(a)

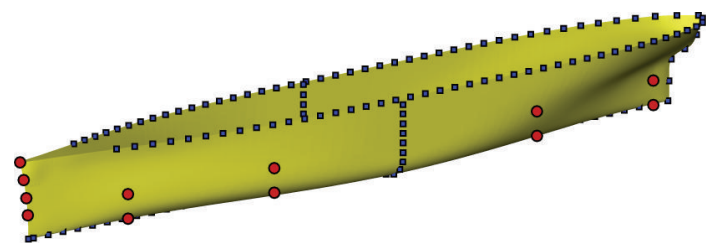

(b)

FIGURE 2: RBF control nodes.

with $f_{j}$ containing the discrete known values of the displacement on the boundary (control nodes) and the additional requirements

$$
\sum_{j=1}^{N} \lambda_{j} p\left(\mathbf{x}_{j}\right)=0, \quad j=1, \ldots, N
$$

The values of the coefficients $\lambda_{j}$ and $c_{j}$ can be obtained by solving the linear system

$$
\left(\begin{array}{l}
f \\
0
\end{array}\right)=\left(\begin{array}{ll}
M & P \\
P^{T} & 0
\end{array}\right)\left(\begin{array}{l}
\lambda \\
c
\end{array}\right)
$$

where $\lambda=\left[\lambda_{1}, \lambda_{2}, \ldots, \lambda_{N}\right], c=\left[c_{1}, c_{2}, c_{3}, c_{4}\right]$, and $f=$ $\left[f_{1}, f_{2}, \ldots, f_{N}\right]^{T}$. The elements of the matrices $M$ and $P$ are defined as

$$
\begin{gathered}
M_{i, j}=\varphi\left(\left|\mathbf{x}_{i}-\mathbf{x}_{j}\right|\right), \quad i, j=1, \ldots, N, \\
P_{i, j}=p_{j}\left(\mathbf{x}_{i}\right), \quad i=1, \ldots, N, j=1, \ldots, 4 .
\end{gathered}
$$

In this study, $x$-coordinate of eight control nodes RBF 18 and $y$-coordinate of four control nodes RBF 9-12 are defined as design variables for modification of the station profile and the stem profile, respectively, as shown in Figure 2. Table 1 summarizes the design variables and their range used to define the new hull form. All values in this table are normalized by length of hull form which is unit. 
2.2. Objective Functions. The present study is focused on the wave drag performance of hull form over a wide speed range. Therefore, the three objective functions (wave drag performance at three different speeds) are defined as follows:

$$
\begin{gathered}
f_{\mathrm{obj}}^{1}=\frac{\left(C_{W}^{d}-C_{W}^{i}\right)}{C_{W}^{i}} \text { at } F_{N}=0.22, \\
f_{\mathrm{obj}}^{2}=\frac{\left(C_{W}^{d}-C_{W}^{i}\right)}{C_{W}^{i}} \text { at } F_{N}=0.27, \\
f_{\mathrm{obj}}^{3}=\frac{\left(C_{W}^{d}-C_{W}^{i}\right)}{C_{W}^{i}} \text { at } F_{N}=0.305,
\end{gathered}
$$

where $F_{N}$ is Froude number. $C_{W}^{i}$ and $C_{W}^{d}$ denote the wave drag coefficient of the initial hull form and the hull form obtained during the optimization process, respectively. The CFD tool for evaluating the wave drag (objective function) is the steady ship flow (SSF), which is based on NeumannMitchell (NM) theory [24]. Even though NM theory is a low fidelity method, the wave drag/total drag from this method shows a fairly good agreement with experimental measurements [25].

\section{Design Exploration Framework}

Design exploration framework suggested in this study consists of multiobjective shape optimization and design knowledge extraction. In the multiobjective shape optimization, design space is explored using multiobjective genetic algorithm and response surface model to find the optimum solutions. In the design knowledge extraction, data mining techniques are applied to obtain the useful design information, which is helpful for understanding of design problem. Figure 3 shows the overall procedure the design exploration framework in this study. The details of each method are explained in the following subsections.

\subsection{Multiobjective Shape Optimization}

3.1.1. Initial Sample Selection. The initial sample points are selected by Latin hypercube sampling (LHS) [26] to construct the response surface model for each objective function. It is desirable to distribute sample points uniformly in the design space. The sample points distributed by LHS satisfied the orthogonal condition, which means that points do not overlap for all design variables. Thus, it is possible to distribute points evenly in the design space with a small number of sample points. In this study, 60 points are selected as initial sample points and wave drag performances of these hull forms are evaluated using SSF code.

3.1.2. Response Surface Model Construction. With the initial sample points selected in the previous step, the response surface model is constructed for each objective function. The response surface model adopted in this study is the Kriging model $[11,13]$, which was developed in the field of spatial statistics and geostatistics. The Kriging predictor is expressed as follows:

$$
\widehat{y}(\mathbf{x})=\widehat{\beta}+\mathbf{r}^{T} \mathbf{R}^{-1}(\mathbf{y}-\mathbf{1} \widehat{\beta})
$$

where $\mathbf{x}=x_{1}, x_{2}, \ldots, x_{m}$ is an $m$-dimensional vector of the design variable, $\mathbf{y}$ is an $n$-dimensional column vector of sampled response data, $\mathbf{1}$ is an $n$-dimensional unit column vector, and $\mathbf{R}$ is the correlation matrix whose $(i, j)$ element is given by

$$
\mathbf{R}\left(\mathbf{x}^{i}, \mathbf{x}^{j}\right)=\exp \left[-\sum_{k=1}^{m} \theta_{k}\left|x_{k}^{i}-x_{k}^{j}\right|^{2}\right], \quad i, j=1, \ldots n .
$$

The correlation vector between $\mathbf{x}$ and the $n$ sampled data is expressed as

$$
\mathbf{r}^{T}(\mathbf{x})=\left[\mathbf{R}\left(\mathbf{x}, \mathbf{x}^{1}\right), \mathbf{R}\left(\mathbf{x}, \mathbf{x}^{2}\right), \ldots, \mathbf{R}\left(\mathbf{x}, \mathbf{x}^{n}\right)\right] .
$$

The $\widehat{\beta}$ can be calculated using the following equation:

$$
\widehat{\beta}=\frac{\mathbf{1}^{T} \mathbf{R}^{-1} \mathbf{y}}{\mathbf{1}^{T} \mathbf{R}^{-1} \mathbf{1}} \text {. }
$$

The unknown parameter, $\theta$, for the Kriging model can be estimated by maximizing the following likelihood function:

$$
\ln \left(\widehat{\beta}, \widehat{\sigma}^{2}, \theta\right)=-\frac{n}{2} \ln \left(\widehat{\sigma}^{2}\right)-\frac{1}{2} \ln (|\mathbf{R}|),
$$

where $\sigma^{2}$ can be calculated as follows:

$$
\widehat{\sigma}^{2}=\frac{(\mathbf{y}-\mathbf{1} \widehat{\beta})^{T} \mathbf{R}(\mathbf{y}-\mathbf{1} \widehat{\beta})}{n} .
$$

Maximization of the likelihood function is an $m$-dimensional unconstrained nonlinear optimization problem. A simple genetic algorithm is used to determine $\theta$.

The accuracy of the predicted value largely depends on the distance from sample points. Intuitively speaking, the closer point $\mathbf{x}$ is to the sample point, the more accurate is the prediction $\widehat{y}(\mathbf{x})$. This intuition is expressed as

$$
s^{2}(\mathbf{x})=\widehat{\sigma}^{2}\left[1-\mathbf{r}^{\prime} \mathbf{R}^{-1} \mathbf{r}+\frac{\left(1-\mathbf{1}^{-\mathbf{1}} \mathbf{r}\right)^{2}}{\mathbf{1}^{\prime} \mathbf{R}^{-\mathbf{1}} \mathbf{1}}\right],
$$

where $s^{2}(\mathbf{x})$ is the mean squared error of the predictor and it indicates the uncertainty of the predicted value.

Once the Kriging model is constructed, cross-validation is performed to validate the accuracy of the model as shown in Figure 4. The objective function values estimated by the Kriging model show good agreement with those estimated by SSF solver.

3.1.3. Design Space Exploration and Additional Sample Points Selection. In the constructed Kriging model, the minimum wave drag hull form (nondominated solutions and Pareto solutions) has been explored using a multiobjective genetic 


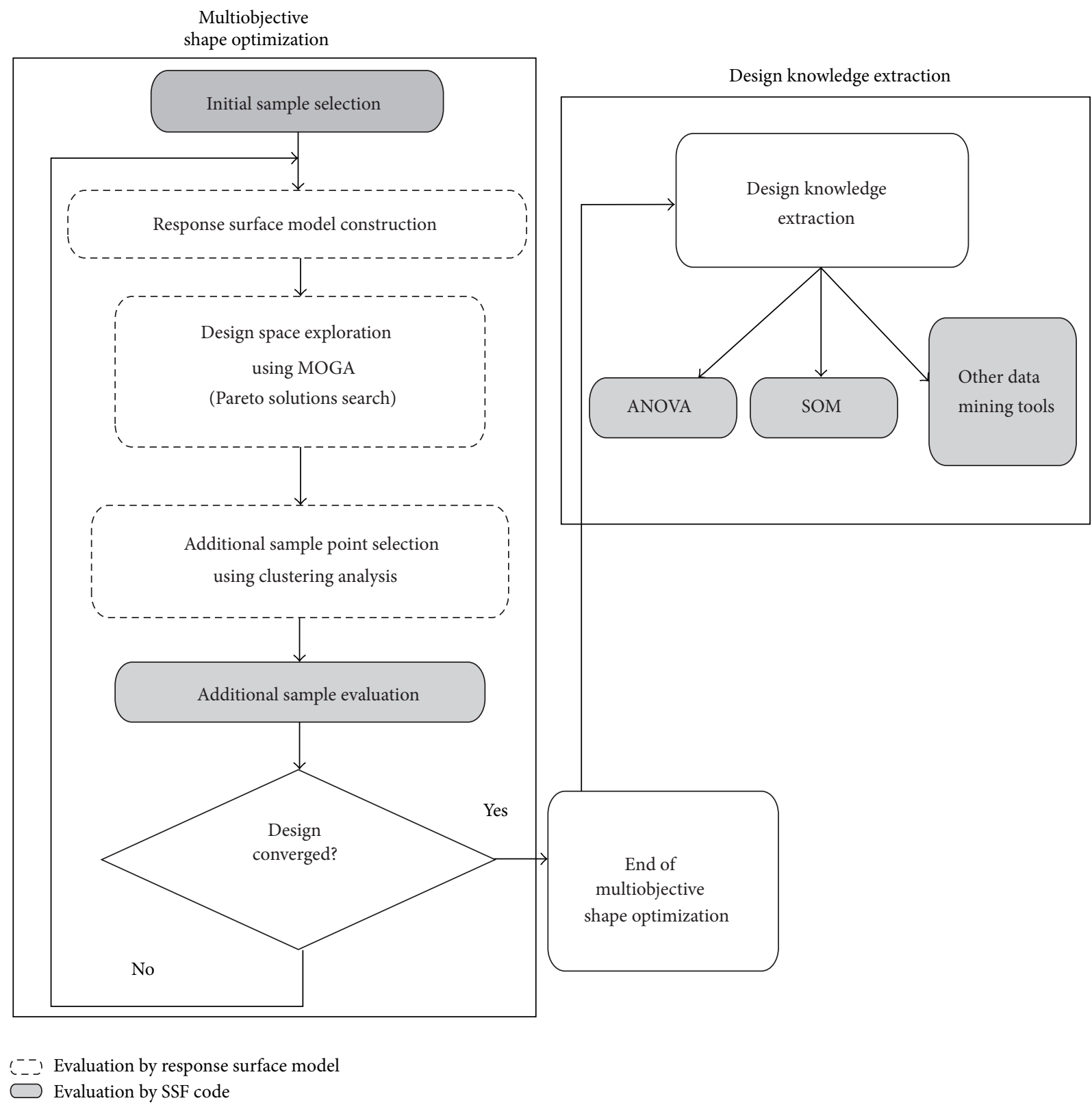

FIGURE 3: Overall procedure of design exploration framework.

algorithm (MOGA). The number of populations and generations of MOGA were 512 and 100, respectively. Figure 5(a) shows the nondominated solutions obtained after MOGA operation in Figure 3. To investigate the performance of these nondominated solutions and select additional sample points, the $K$-means clustering method $[27,28]$ is applied to the nondominated solution obtained. The $K$-means method subgroups the nondominated solutions based on the similarity of three objective function values of solutions. Figure 5(b) shows the nondominated solution after clustering analysis. The solutions with the same color have similar objective function values. The center of each cluster is selected as representative of cluster and the wave drag of these points is evaluated by SSF to check the performance. Then, these points are used as additional sample points for the update of Krignig model. This process is iterated until the Pareto front of the nondominated solutions is converged.

3.2. Design Knowledge Extraction. Two data mining techniques $[16,29]$, analysis of variance (ANOVA) and self-organizing map (SOM), are applied to the results of multiobjective shape optimization to extract knowledge of hull design. Details of these will be introduced in following sections.

3.2.1. Analysis of Variance (ANOVA). ANOVA is a commonly used statistical analysis method, which uses the variance model to show the effects of each variable on the function. The 

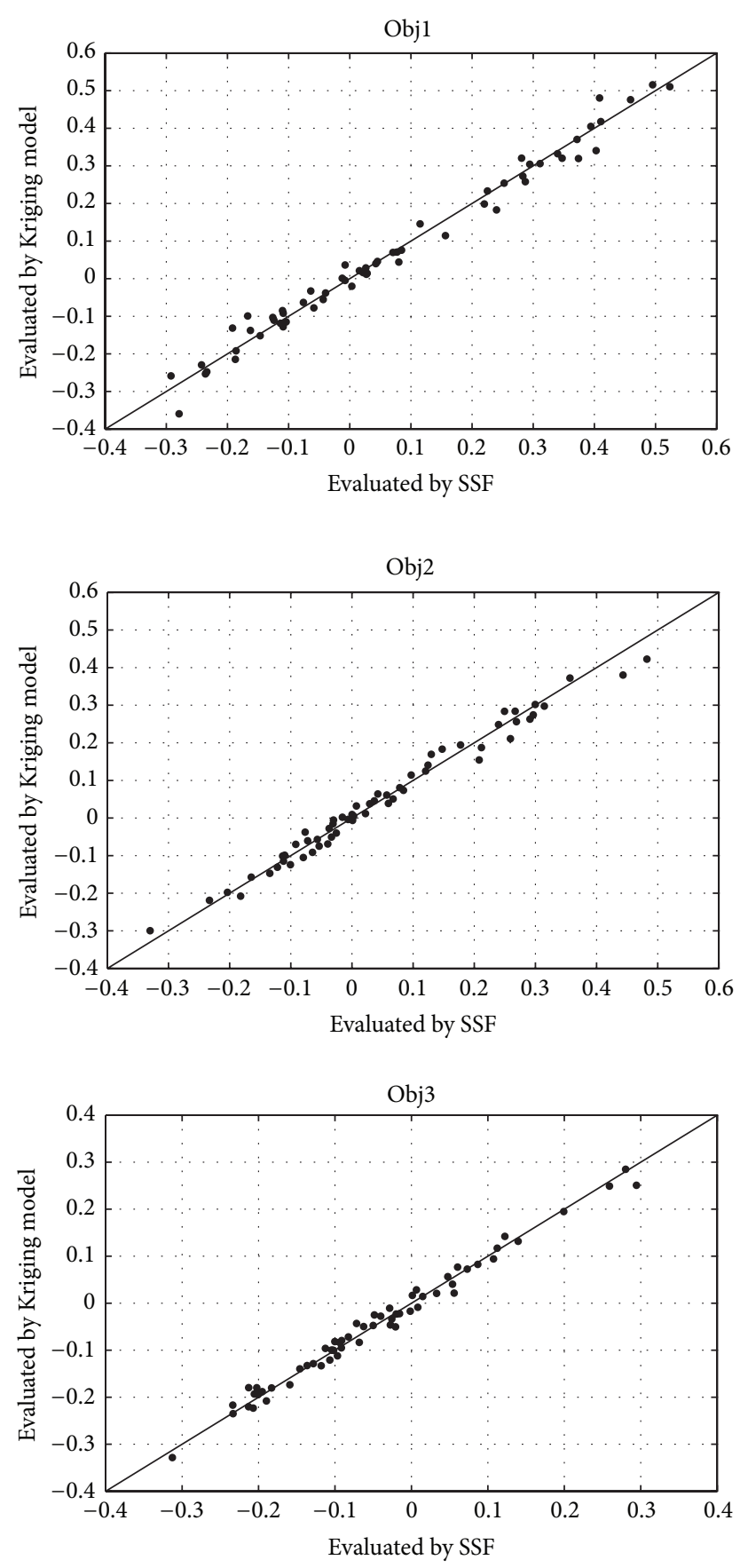

FIgURE 4: Cross validation.

ANOVA employed in this study decomposed the total variance of the model into the variance due to each design variable on the Kriging response surface model used in the multiobjective shape optimization.

The total mean $\left(\widehat{\mu}_{\text {total }}\right)$ and the variance $\left(\widehat{\sigma}_{\text {total }}^{2}\right)$ of the model are as follows:

$$
\begin{gathered}
\widehat{\mu}_{\text {total }} \equiv \int \cdots \int \widehat{y}\left(x_{1}, \ldots, x_{m}\right) d x_{1} \cdots d x_{m}, \\
\widehat{\sigma}_{\text {total }}^{2} \equiv \int \ldots \int\left[\hat{y}\left(x_{1}, \ldots, x_{m}\right)-\widehat{\mu}_{\text {total }}\right]^{2} d x_{1} \cdots d x_{m} .
\end{gathered}
$$

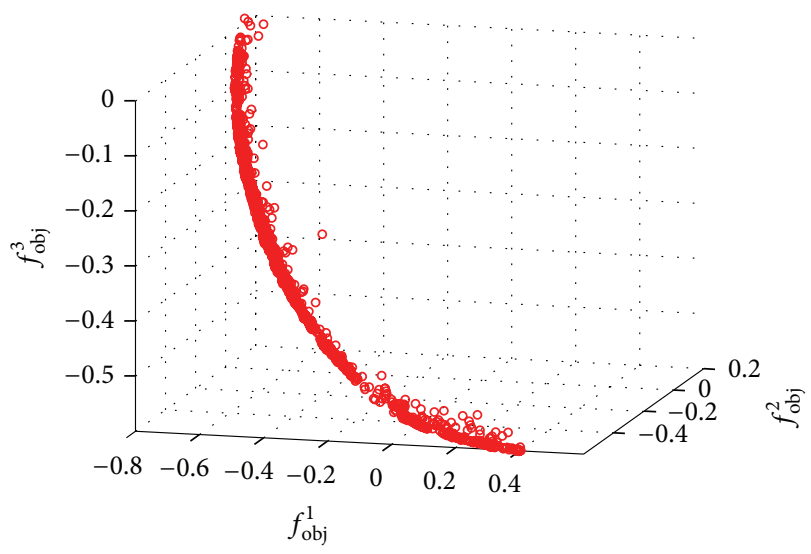

(a) Nondominated solutions

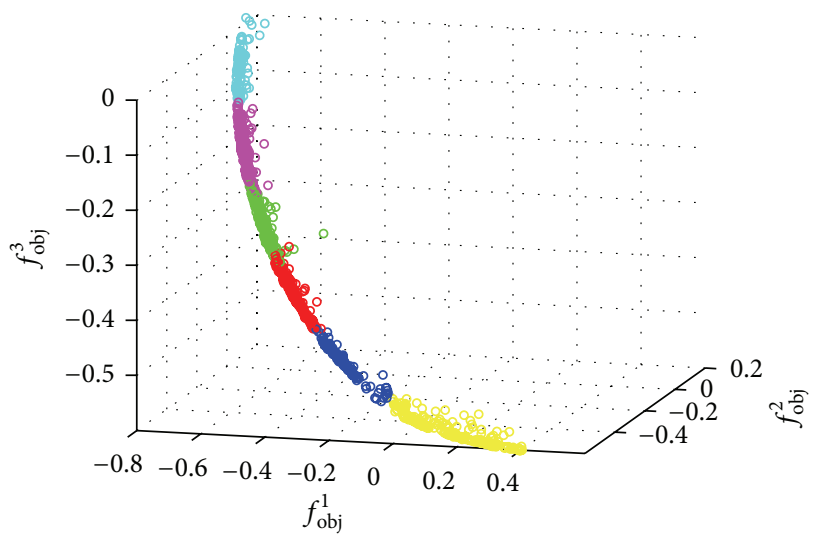

(b) Nondominated solutions after clustering

FIGURE 5: Nondominated solutions obtained from MOGA.

The main effect $\left(\widehat{\mu}_{i}\left(x_{i}\right)\right)$ and variance $\left(\widehat{\sigma}_{i}^{2}\left(x_{i}\right)\right)$ of variable $x_{i}$ can be given as

$$
\begin{gathered}
\widehat{\mu}_{i}\left(x_{i}\right) \\
\equiv \int \cdots \int \widehat{y}\left(x_{1}, \ldots, x_{m}\right) d x_{1} \cdots d x_{i-1} d x_{i+1} \cdots d x_{m}-\widehat{\mu}_{\text {total }}, \\
\widehat{\sigma}_{i}^{2}\left(x_{i}\right)=\int\left[\widehat{\mu}_{i}\left(x_{i}\right)\right]^{2} d x_{i} .
\end{gathered}
$$

The proportion of the variance due to design variable $x_{i}$ to total variance of the model can be calculated by dividing (18) by (20). This value indicated the effect of design variable $x_{i}$ on the objective function:

$$
\frac{\widehat{\sigma}_{i}^{2}}{\widehat{\sigma}_{\text {total }}^{2}}=\frac{\int\left[\widehat{\mu}_{i}\left(x_{i}\right)\right]^{2} d x_{i}}{\int \cdots \int\left[\widehat{y}\left(x_{1}, \ldots, x_{m}\right)-\widehat{\mu}_{\text {total }}\right]^{2} d x_{1} \cdots d x_{m}} .
$$

Thus, if this value is large, $x_{i}$ has a large effect on the objective function. If not, $x_{i}$ can be considered as less important design variable for the objective function.

3.2.2. Self-Organizing Map (SOM). SOM is an unsupervised neural network technique that classifies, organizes, and visualizes large data sets. SOM is a nonlinear projection algorithm 


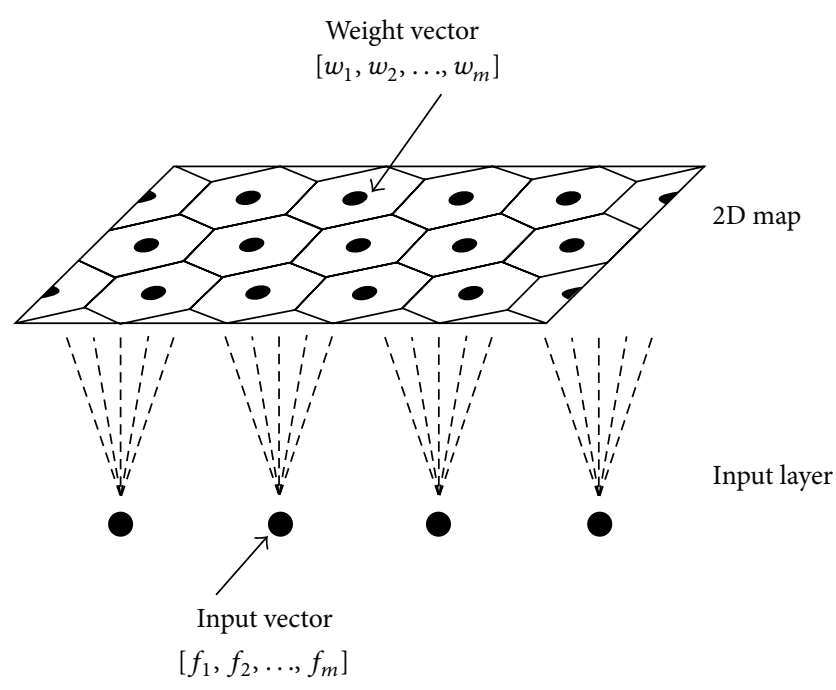

FIGURE 6: Self-organizing map (SOM).

from high- to low-dimensional space. This projection is based on self-organization of a low-dimensional array of neurons. In the projection algorithm, the weights between the input vector (input data) and the array of neurons are adjusted to represent features of the high-dimensional original data on the low-dimensional map. The closer two-input data are in the original space, the closer the response of two neighboring neurons is in the low-dimensional space. Thus, SOM reduces the dimension of input data, while preserving their features.

A neuron used in SOM is associated with weight vector $\mathbf{w}_{i}=\left[w_{i 1}, w_{i 2}, \ldots, w_{i m}\right](i=1, \ldots, N)$ where $m$ is equal to the dimension of the input vector and $N$ is the number of neurons. Each neuron is connected to adjacent neurons by a neighborhood relation and usually forms a two-dimensional hexagonal topology as shown in Figure 6.

The learning algorithm of SOM [17] is started with finding the best-matching unit $\left(\mathbf{w}_{c}\right)$, which is the closest to the input vector $\mathbf{f}=\left[f_{1}, \ldots, f_{m}\right]$ as follows:

$$
\left\|\mathbf{f}-\mathbf{w}_{c}\right\|=\min \left\|\mathbf{f}-\mathbf{w}_{k}\right\| \quad(k=1, \ldots, N) .
$$

Then, the weight vector of the best-matching unit and its neighbors is adjusted to make its location much closer to the input vector. Repeating this learning algorithm, the weight vectors become smooth not only locally but also globally. As a result, the sequence of close vectors in the original space results in a sequence of corresponding neighboring neurons in the two-dimensional map.

\section{Results}

4.1. Results of Shape Optimization. In this study, the multiobjective shape optimization process shown in Figure 3 is iterated three times. Figure 7 shows the initial sample points used to construct the Kriging model and the nondominated solutions obtained after three iterations of the multiobjective shape optimization. It can be confirmed that the obj1-obj2 and obj1-obj3 show a strong trade-off relation, while obj2-obj3

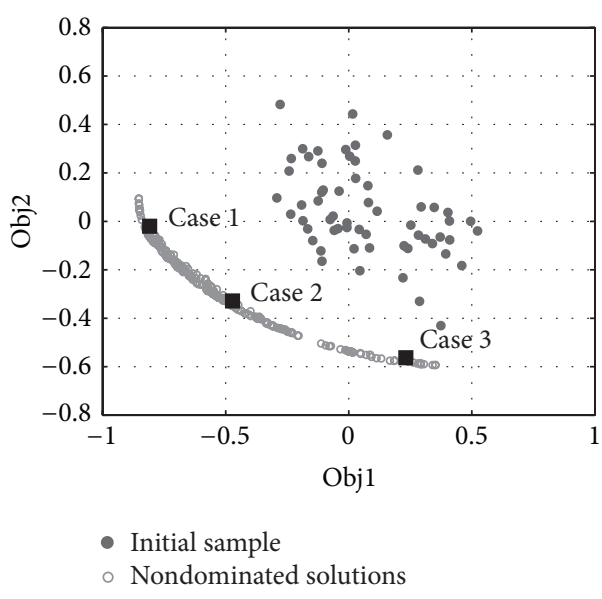

(a) Obj1 versus Obj2

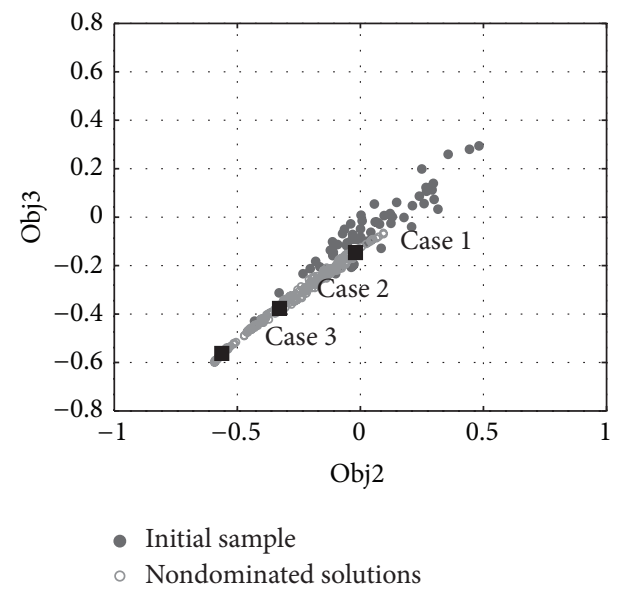

(b) Obj2 versus Obj3

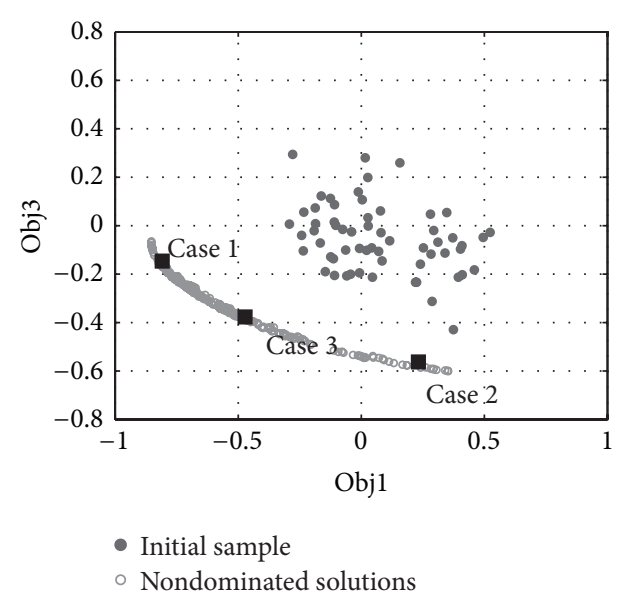

(c) Obj1 versus Obj3

FIgURE 7: Results of shape optimization.

shows a linear relation. This implies that the performance in terms of wave drag at the low-speed design speed $\left(F_{N}=0.22\right)$ is opposed with those at both intermediate $\left(F_{N}=0.27\right)$ and high design speeds $\left(F_{N}=0.305\right)$ and that the performance in terms of wave drag at intermediate design speed is consistent with that at high design speed. The total computational time 
taken to obtain the nondominated solutions was 20 min on a PC (2.66 GHz Intel Xeon, 4 Gbytes RAM, Mac OS). If this shape optimization had been performed without the Kriging model, even though the computational time of SSF for one case is 10 seconds, the total computational time taken to obtain the solutions would be about 18 days ( 10 seconds $\times 512$ populations $\times 100$ generations $\times 3$ iterations).

Among the nondominated solutions, three typical optimum solutions-Case 1, Case 2, and Case 3-are selected to investigate wave drag performance over a wide speed range as shown in Figure 7. Case 1 is the solution with low wave drag performance at low design speed. Case 2 is the solution with low wave drag performance at high design speed. Case 3 is a compromise solution between Case 1 and Case 2. Figure 8 compares the wave and total drag coefficient $\left(C_{W}\right.$ and $C_{T}$ ) between the initial and each of these three solutions. Case 1 shows the best drag performance in the low-speed regime, while Case 2 shows the best drag performance in the high-speed regime. As expected, Case 3 shows intermediate drag performance at all speed ranges. Table 2 shows the percentages of wave and total drag coefficient variation from those of baseline configuration. The friction drag is calculated according to the International Towing Tank Conference (ITTC) model-ship correlation line [30]:

$$
C_{F}=\frac{0.075}{\left(\log _{10} \operatorname{Re}-2\right)^{2}},
$$

where Re is Reynolds number for the respective temperature.

Table 3 displays variations in geometric properties. Even though each optimum hull exhibits very small changes in the wetted surface area $\left(S_{\text {wet }}\right.$ : the amount of area of hull that is under water) and displacement $(\nabla$ : the volume of fluid displaced by hull), the wave performance of these hull forms is improved very much.

\subsection{Results of Design Knowledge Mining}

4.2.1. Analysis of Variance (ANOVA). To investigate the effects of design variables on each objective function, ANOVA is applied to the results of shape optimization. According to results, DV10 and DV11 have a large effect on $f_{\text {obj }}^{1}$, while DV8 and DV12 have large effects on $f_{\text {obj }}^{2}$ and $f_{\text {obj }}^{3}$ as shown in Figure 9. $f_{\mathrm{obj}}^{1}$ is the drag performance at relatively low speed, and $f_{\text {obj }}^{2}$ and $f_{\text {obj }}^{3}$ are those at high speed.

Figure 10 compares the profile plan of the initial hull form with that of Case 1 (low-speed hull form) and Case 2, which has good drag performance at low and high speeds, respectively. Compared with initial hull form, Case 1 has a slightly wider section shape of the lower part (near DV11) and a narrower waterline in the bow part (near DV10), while Case 2 has a narrower waterline near DV12 and DV8. According to the general knowledge about ship design, hull form with a wide section shape shows the good wave drag performance at the low speed while that with a narrow section shape shows the good wave drag performance at the high speed. Thus, the information (design knowledge) obtained through ANOVA corresponds to the general knowledge about ship design.

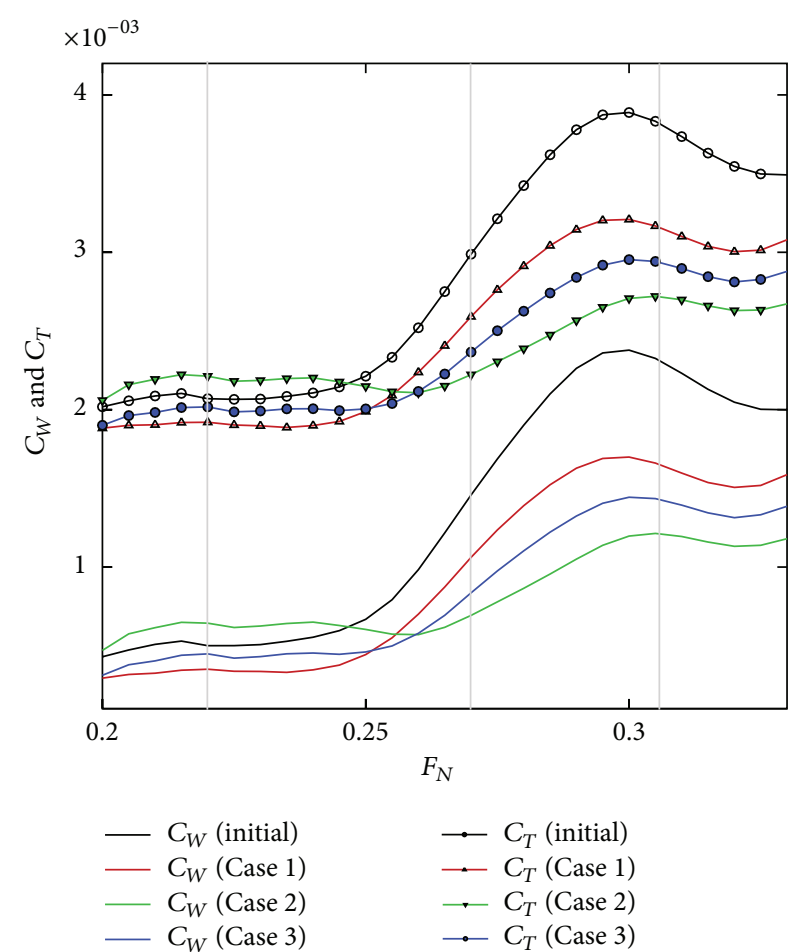

FIGURE 8: Comparison of the wave and total drag coefficient for the initial and three typical solutions.

TABle 2: Percentage of wave and total drag coefficient variation.

\begin{tabular}{cccccccc}
\hline & $\Delta C_{W}^{1}$ & $\Delta C_{W}^{2}$ & $\Delta C_{W}^{3}$ & $\Delta C_{T}^{1}$ & $\Delta C_{T}^{2}$ & $\Delta C_{T}^{3}$ \\
\multicolumn{5}{c}{ Wave drag (\%) } & \multicolumn{3}{c}{ Total drag (\%) } \\
\hline Case 1 & -70.90 & -0.65 & -14.62 & -17.14 & -0.32 & -8.87 \\
Case 2 & 23.20 & -56.60 & -56.60 & 5.60 & -27.47 & -34.14 \\
Case 3 & -43.17 & -32.18 & -32.18 & -10.44 & -15.70 & -22.61 \\
\hline
\end{tabular}

TABLE 3: Variation of wetted surface area $\left(S_{\text {wet }}\right)$ and displacement $(\nabla)$.

\begin{tabular}{lcc}
\hline & $S_{\text {wet }}(\%)$ & $\nabla(\%)$ \\
\hline Case 1 & 0.82 & -0.04 \\
Case 2 & 0.60 & -0.27 \\
Case 3 & 0.16 & -0.19 \\
\hline
\end{tabular}

4.2.2. Self-Organizing Map (SOM). To identify the parameter controlling the trade-off between low- and high-speed geometries, SOM is applied to the nondominated solutions obtained from shape optimization. The number of solutions used for SOM is 1024. Figure 11 shows the SOM obtained after clustering based on the objective functions. As described in Section 3.2.2, SOM consists of a large number of neurons that have a hexagonal topology and the same dimensional vector value as the input data. Thus, SOM can be colored by each objective function value and design variable value. Figure 12 shows the SOMs colored by each objective function value.

The solutions on the left-hand side have a preferable $f_{\text {obj }}^{1}$ and unfavorable $f_{\mathrm{obj}}^{2}$ and $f_{\mathrm{obj}}^{3}$, while the solutions on the 


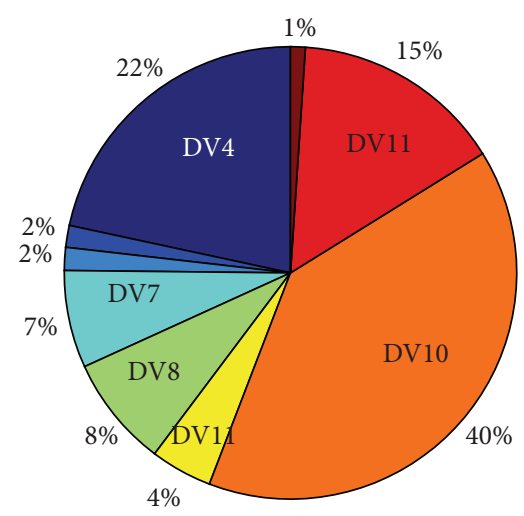

(a) $f_{\text {obj }}^{1}$

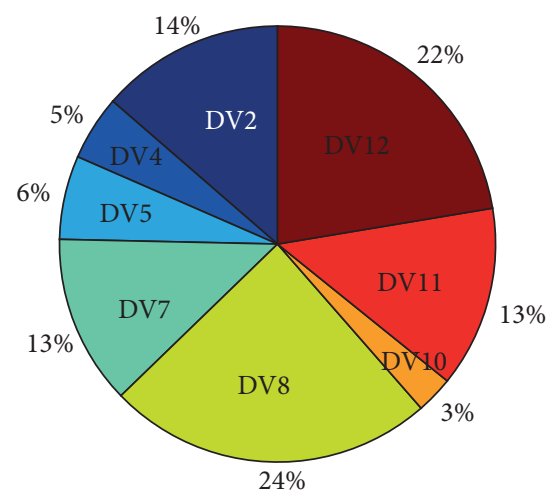

(b) $f_{\text {obj }}^{2}$

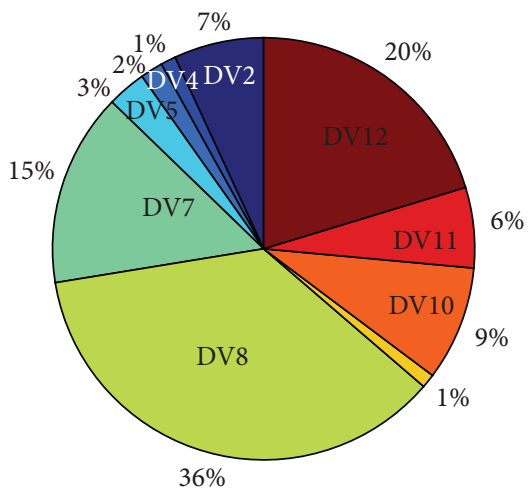

(c) $f_{\text {obj }}^{3}$

Figure 9: Results of ANOVA.

right-hand side have an unfavorable $f_{\mathrm{obj}}^{1}$ and preferable $f_{\mathrm{obj}}^{2}$ and $f_{\text {obj. }}^{3}$. Thus, it can be seen that there is a severe trade-off between low-speed wave $\operatorname{drag}\left(f_{\mathrm{obj}}^{1}\right)$ and high-speed wave drag $\left(f_{\mathrm{obj}}^{2}\right.$ and $\left.f_{\mathrm{obj}}^{3}\right)$ performances. This trade-off was also confirmed in Figure 7. To identify the relation between objective functions and design variables, the SOM is also colored by each design variable value.

Figure 13 shows the SOMs colored by DV4, DV7, DV8, DV10, DV11, and DV12, which have relatively large effects on objective functions according to the ANOVA results in Figure 9.

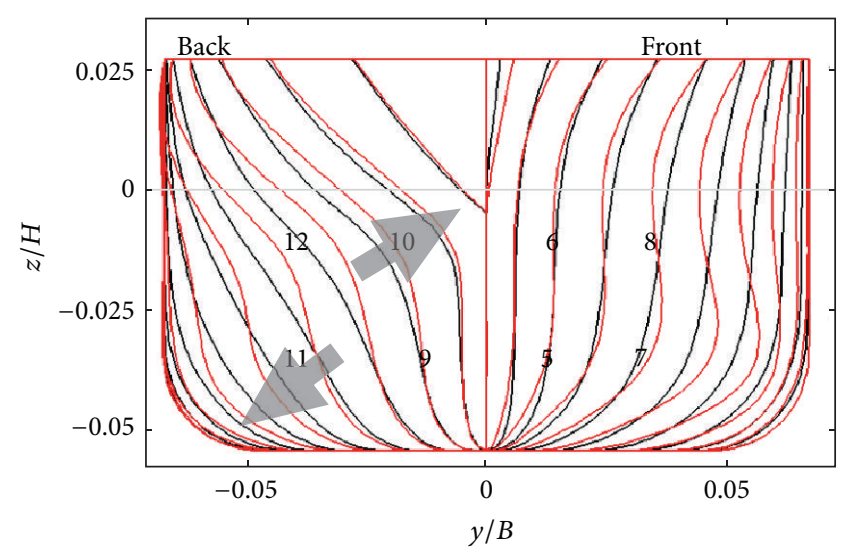

(a) Case 1 (red line) and initial hull form (black line)

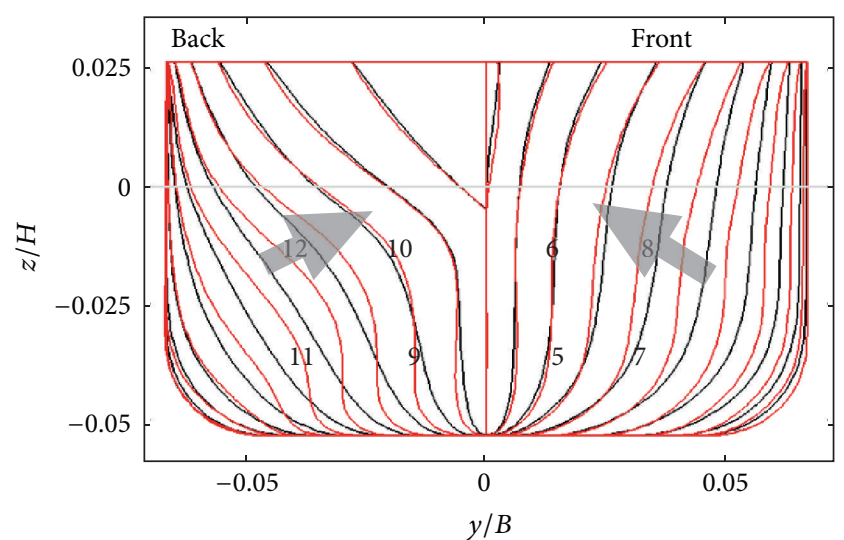

(b) Case 2 (red line) and initial hull form (black line)

FIGURE 10: Comparison of the profile plan.

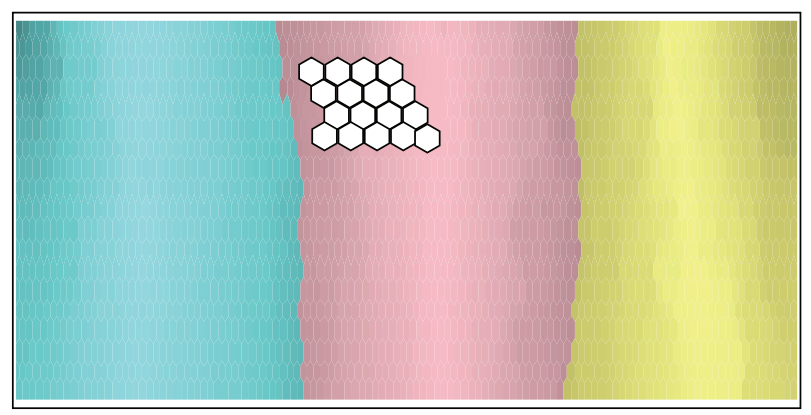

FIGURE 11: SOM obtained after clustering based on the similarity of objective functions.

The solutions on the left-hand side have relatively large values of DV4, DV7, DV8, and DV11, while those of the right-hand side have relatively small values. Thus, the lowspeed geometry should have a wider section shape, while the high-speed geometry should have a narrower section shape. This corresponds to general knowledge of ship hull design. Although DV10 and DV12 have large effects on objective functions according to ANOVA, the values of both DV10 and DV12 are always small regardless of low- or high-speed geometry. It seems that small DV10 and DV12 (narrower 


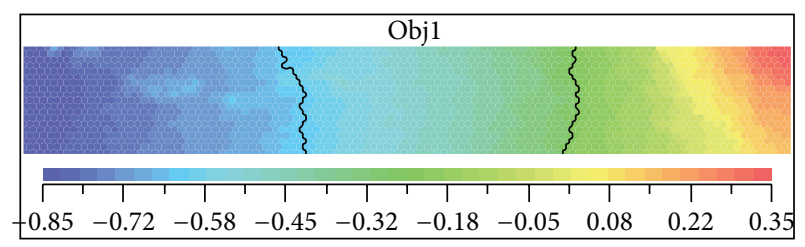

(a) $f_{\text {obj }}^{1}$

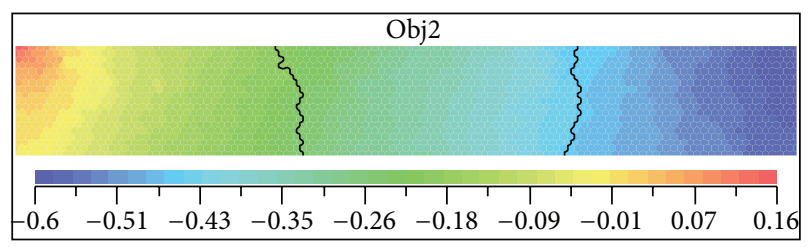

(b) $f_{\text {obj }}^{2}$

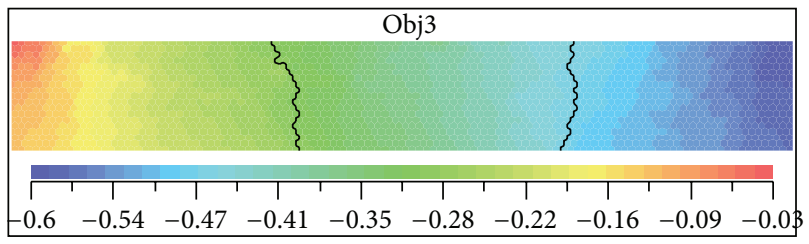

(c) $f_{\text {obj }}^{3}$

FIGURE 12: SOMs colored by objective functions.

waterline in the bow region) are necessary conditions for a nondominated solution.

Thus, the design knowledge obtained through the present data mining can be summarized as follows (Figure 14).

(1) A narrower waterline in the bow region (small DV10 and DV11) is a necessary condition for a nondominated solution.

(2) A wider section shape (large DV4, DV7, DV8, and DV12) is preferable for low-speed performance, while a narrower section shape (small DV4, DV7, DV8, and DV12) is necessary for high-speed performance.

\section{Conclusion and Future Works}

In this study, a high-efficiency design exploration framework consisting of multiobjective shape optimization and design knowledge extraction has been suggested for hull form design. In the multiobjective shape optimization, a multiobjective genetic algorithm (MOGA) using the response surface methodology was adopted. The present framework has been applied to hull form optimization exploring the minimum wave drag configuration over a wide range of speeds. The results show that the present method markedly reduces the design period.

In design knowledge extraction, two data mining techniques, functional analysis of variance (ANOVA) and selforganizing map (SOM), are applied to acquire useful design knowledge regarding hull form. The results of data mining revealed the design variables controlling wave drag performance and its corresponding geometric features.

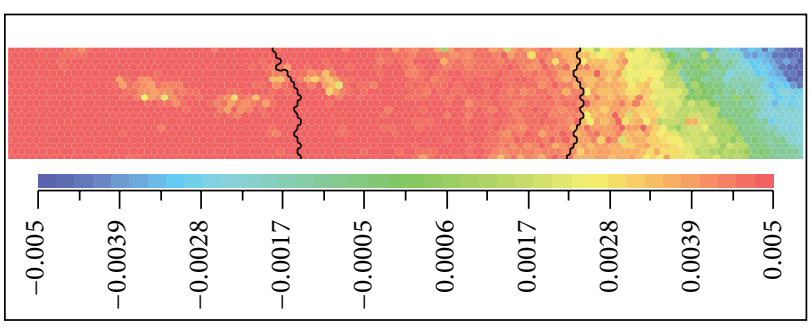

(a) DV4

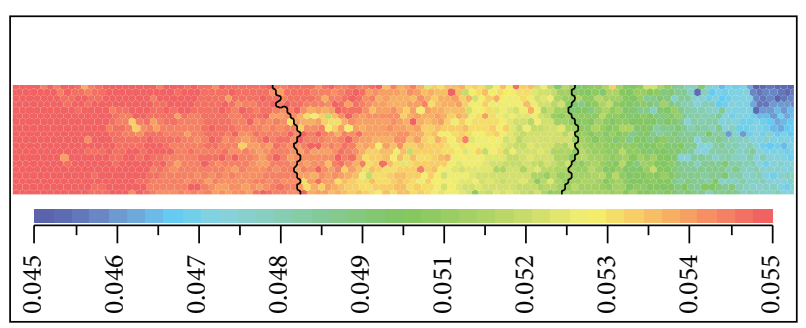

(b) DV7

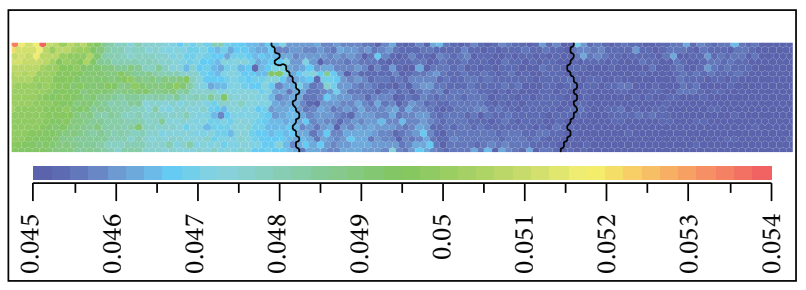

(c) DV8

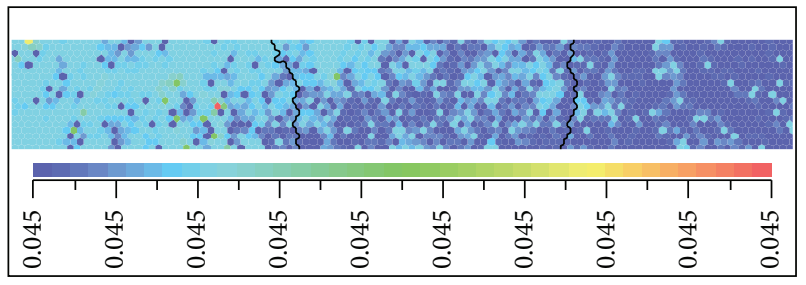

(d) DV10

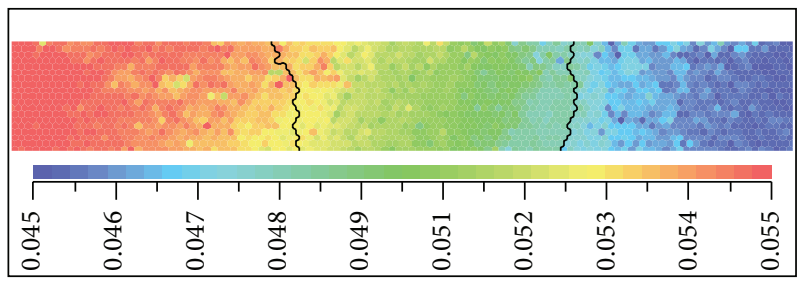

(e) DV11

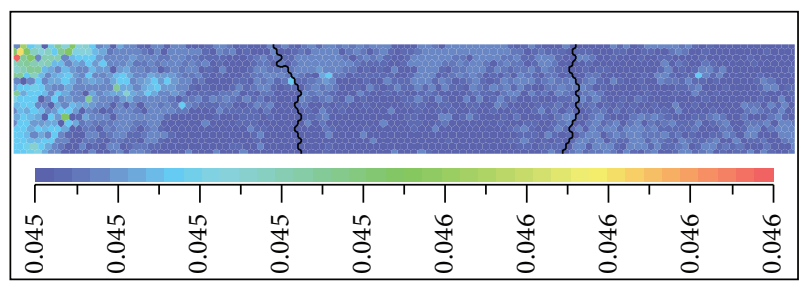

(f) DV12

FIGURE 13: SOMs colored by design variables. 


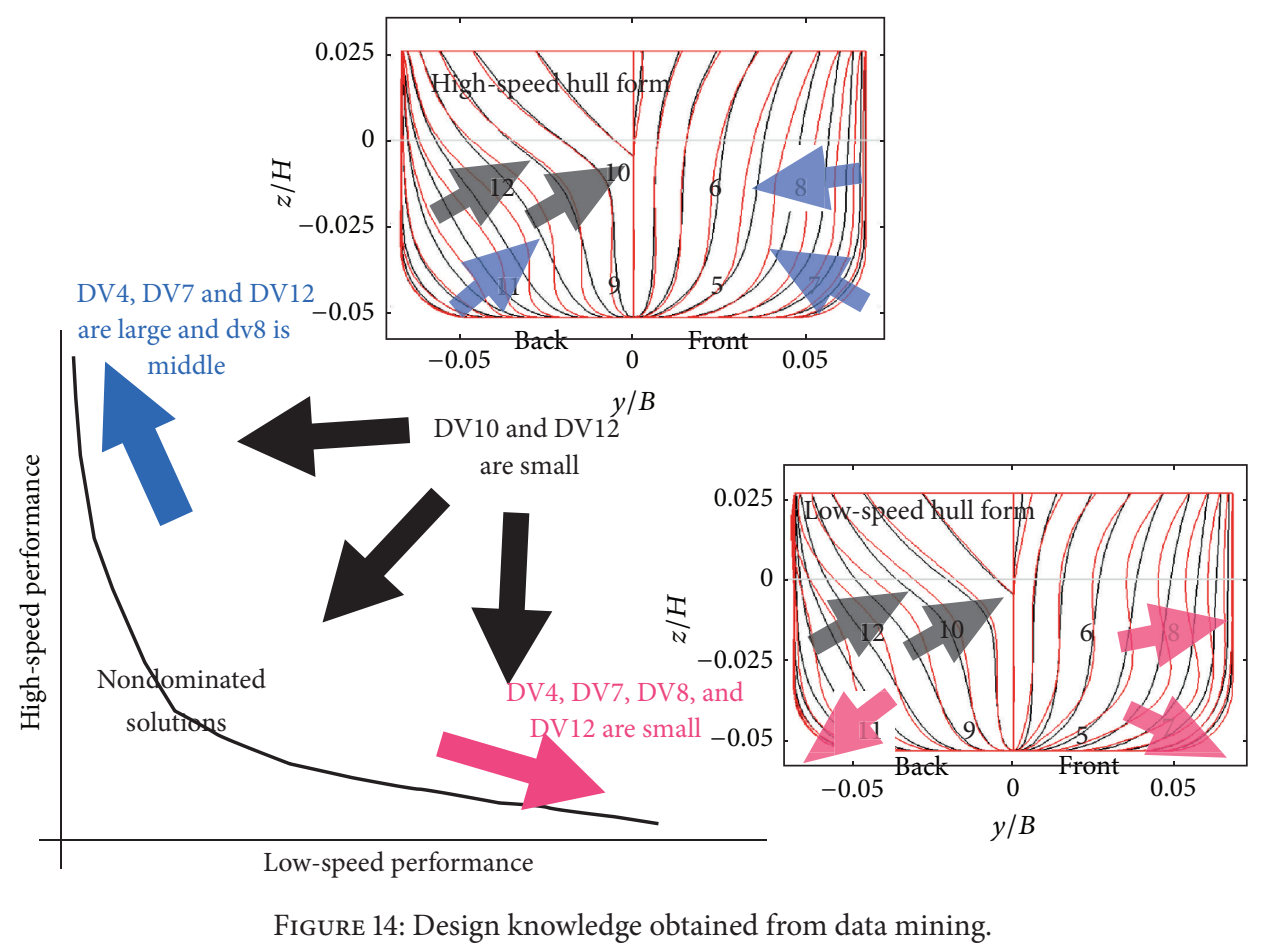

As a future work, the present method will be applied to the nonlinear dynamics of ocean wave, local sea level fluctuation $[31,32]$, and seakeeping problems for the further validation.

\section{References}

[1] D. E. Goldberg, Genetic Algorithms in Search, Optimization and Machine Learning, Kluwer Academic Publishers, Boston, Mass, USA, 1989.

[2] K. Deb, Multi-objective optimization using evolutionary algorithms, John Wiley \& Sons, Chichester, UK, 2001.

[3] J. Kennedy and R. Eberhart, "Particle swarm optimization," in Proceedings of the IEEE International Conference on Neural Networks. Part 1 (of 6), pp. 1942-1948, December 1995.

[4] T. W. Lowe and J. Steel, "Conceptual hull design using a genetic algorithm," Journal of Ship Research, vol. 47, no. 3, pp. 222-236, 2003.

[5] Y. Tahara, S. Tohyama, and T. Katsui, "CFD-based multi-objective optimization method for ship design," International Journal for Numerical Methods in Fluids, vol. 52, no. 5, pp. 499-527, 2006.

[6] Y. Tahara, D. Peri, E. F. Campana, and F. Stern, "Computational fluid dynamics-based multiobjective optimization of a surface combatant using a global optimization method," Journal of Marine Science and Technology, vol. 13, no. 2, pp. 95-116, 2008.

[7] Y. Tahara, T. Hino, M. Kandasamy, W. He, and F. Stern, "CFDbased multiobjective optimization of waterjet propelled high speed ships," in Proceedings of the 11th International Conference on Fast Sea Transportation (FAST '11), Honolulu, Hawaii, 2011.

[8] M. A. Gammon, "Optimization of fishing vessels using a multiobjective genetic algorithm," Ocean Engineering, vol. 38, no. 10, pp. 1054-1064, 2011.

[9] J. T. Knight, F. T. Zahradka, D. J. Singer, and M. D. Collette, "Multi-objective particle swarm optimization of a planing craft with uncertanity," in Proceedings of the 11th International Conference on Fast Sea Transportation (FAST '11), Honolulu, Hawaii, 2011.

[10] G. E. P. Box, W. G. Hunter, and J. S. Hunter, Statistics for Experimenters, John Wiley \& Sons, New York, NY, USA, 1978.

[11] S. Jeong, M. Murayama, and K. Yamamoto, "Efficient optimization design method using kriging model," Journal of Aircraft, vol. 42, no. 2, pp. 413-420, 2005.

[12] K. Sugimura, S. Obayashi, and S. Jeong, "Multi-objective optimization and design rule mining for an aerodynamically efficient and stable centrifugal impeller with a vaned diffuser," Engineering Optimization, vol. 42, no. 3, pp. 271-293, 2010.

[13] D. R. Jones, M. Schonlau, and W. J. Welch, "Efficient global optimization of expensive black-box functions," Journal of Global Optimization, vol. 13, no. 4, pp. 455-492, 1998.

[14] O. Schabenberger and C. A. Cotway, Statistical Methods For Spatial Data Analysis, New York, NY, USA, Champmand and Hall/CRC, 1st edition, 2004.

[15] T. C. Bailey and W. J. Krzanowski, "An overview of approaches to the analysis and modelling of multivariate geostatistical data," Mathematical Geosciences, vol. 44, no. 4, pp. 381-383, 2012.

[16] S. Jeong, K. Chiba, and S. Obayashi, "Data mining for aerodynamic design space," Journal of Aerospace Computing, Information and Communication, vol. 2, no. 11, pp. 452-469, 2005.

[17] T. Kohonen, Self-Organizing Maps, vol. 30, Springer, Berlin, Germany, 1995.

[18] F. H. Todd and F. X. Forest, A Proposed New Basis For the Design of Single New Screw MerchAnt Ship Forms and a Standard Series of Lines, Transactions of S.N.A.M.E., 1951.

[19] F. H. Todd, Some Further Experiments on Single Screw Merchant Ship Forms-Series 60, Transactions of S.N.A.M.E., 1953.

[20] H. Lackenby, On the Systematic Geometrical Variation of Ship Forms, vol. 92, Transaction of Royal Institute of Naval Architects, 1950 . 
[21] H. Y. Kim, C. Yang, and F. Noblesse, "Hull form optimization of reduced resistance and improved seakeeping via practical design-oriented CFD tool," in Proceedings of the Grand Challenges in Modeling and Simulation Conference, pp. 375-385, 2010.

[22] H. Y. Kim, C. Yang, and H. H. Chun, "A combined local and global hull form modification for hydrodynamic optimization," in Proceedings of the 28th Symposium on Naval Hydrodynamics, Pasadena, CA, USA, 2010.

[23] A. de Boer, M. S. van der Schoot, and H. Bijl, "Mesh deformation based on radial basis function interpolation," Computers and Structures, vol. 85, no. 11-14, pp. 784-795, 2007.

[24] C. Yang, H. Y. Kim, G. Delhommeau, and F. Noblesse, "The Neumann-Kelvin and Neumann-Michell linear models of steady flow about a ship," in Proceedings of the 12th International Congress of the International Maritime Association of the Mediterranean (IMAM '07), pp. 129-136, Varna, Bulgaria, September 2007.

[25] C. Yang, H. Y. Kim, and F. Noblesse, "A practical method for evaluating steady flow about a ship," in Proceedings of the 7th International Conference on Fast Sea Transportation, 2007.

[26] M. D. McKay, R. J. Beckman, and W. J. Conover, "A comparison of three methods for selecting values of input variables in the analysis of output from a computer code," Technometrics, vol. 21, no. 2, pp. 239-245, 1979.

[27] A. K. Jain, M. N. Murty, and P. J. Flynn, "Data clustering: a review," ACM Computing Surveys, vol. 31, no. 3, pp. 316-323, 1999.

[28] P. J. Rousseeuw, "Silhouettes: a graphical aid to the interpretation and validation of cluster analysis," Journal of Computational and Applied Mathematics, vol. 20, pp. 53-65, 1987.

[29] S. Jeong and K. Shimoyama, "Review of data mining for multidisciplinary design optimization," Proceedings of the Institution of Mechanical Engineers G, vol. 225, no. 5, pp. 469-479, 2011.

[30] ITTC, "1978 performance prediction method," ITTC Recommended Procedures and Guideline, Procedure 7. 5-02-01-03, Revision 00, 2002.

[31] N. Su, Z.-G. Yu, V. Anh, and K. Bajracharya, "Fractal tidal waves in coastal aquifers induced both anthropogenically and naturally," Environmental Modelling and Software, vol. 19, no. 12, pp. 1125-1130, 2004.

[32] M. Li, C. Cattani, and S.-Y. Chen, "Viewing sea level by a onedimensional random function with long memory," Mathematical Problems in Engineering, vol. 2011, Article ID 654284, 13 pages, 2011. 


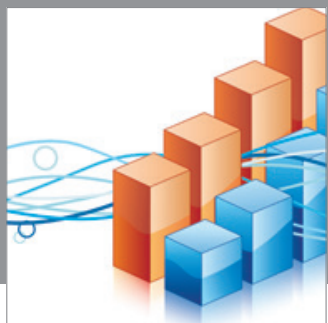

Advances in

Operations Research

mansans

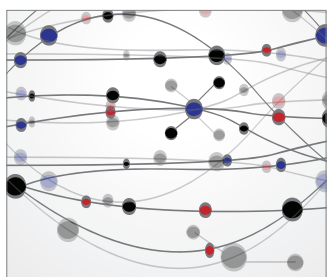

The Scientific World Journal
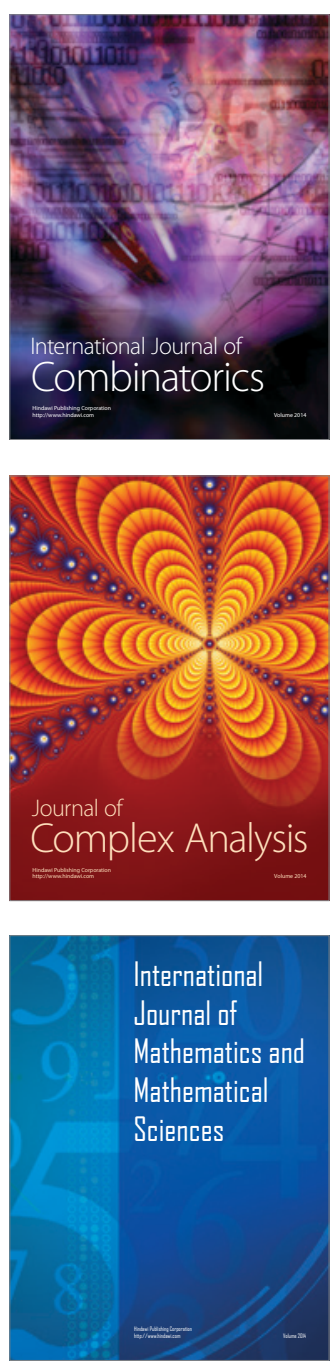
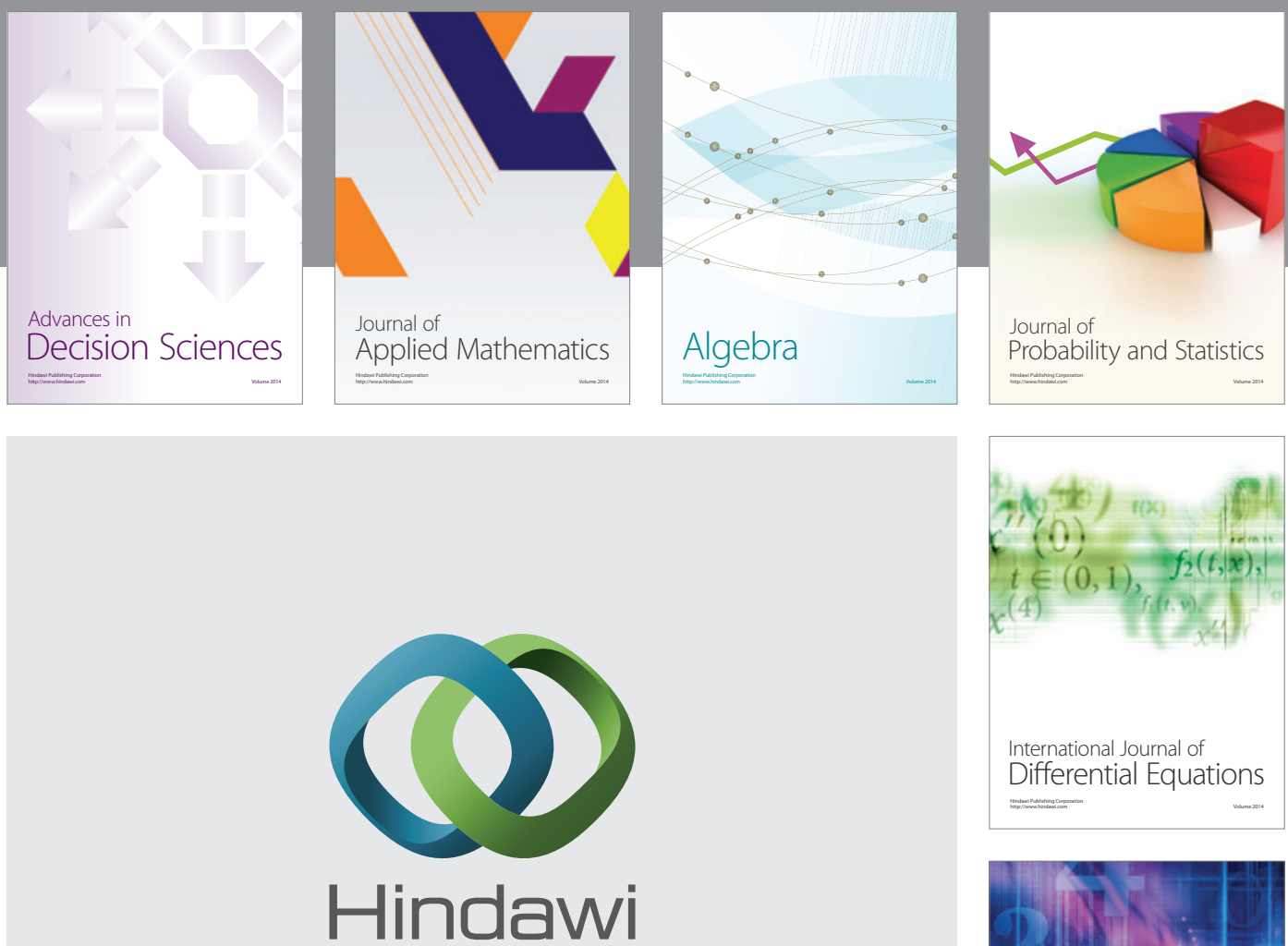

Submit your manuscripts at http://www.hindawi.com
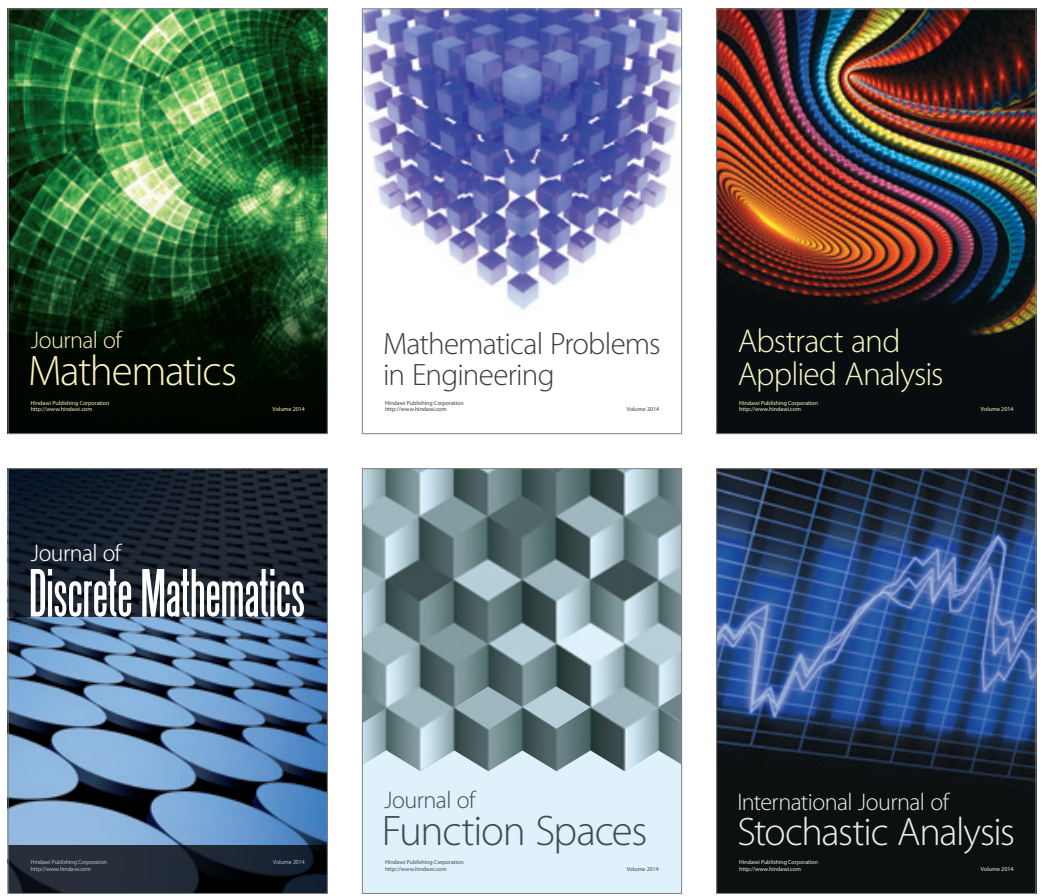

Journal of

Function Spaces

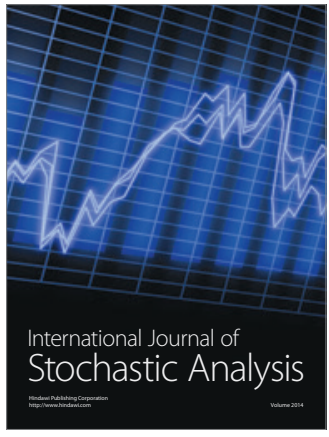

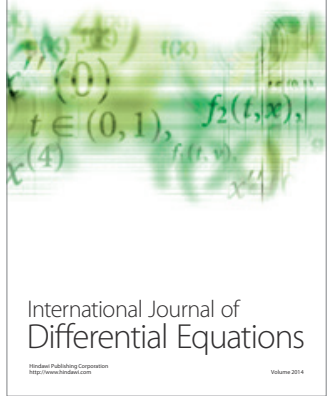
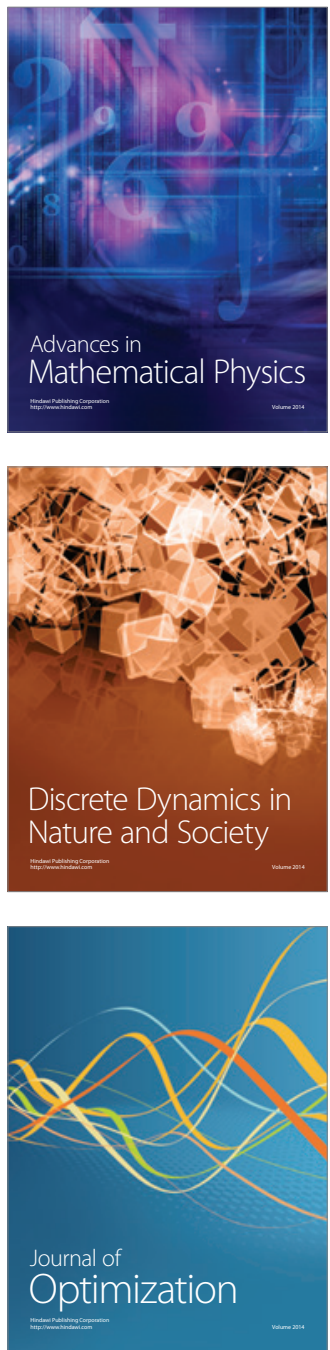